\title{
Impact of Electron-Impurity Scattering on the Spin Relaxation Time in Graphene: A First-Principles Study
}

\author{
Dmitry V. Fedorov, ${ }^{1,2, *}$ Martin Gradhand, ${ }^{3,1}$ Sergey Ostanin, ${ }^{1}$ Igor V. Maznichenko, ${ }^{2}$ \\ Arthur Ernst, ${ }^{1,2}$ Jaroslav Fabian, ${ }^{4}$ and Ingrid Mertig ${ }^{2,1}$ \\ ${ }^{1}$ Max Planck Institute of Microstructure Physics, Weinberg 2, 06120 Halle, Germany \\ ${ }^{2}$ Institute of Physics, Martin Luther University Halle-Wittenberg, 06099 Halle, Germany \\ ${ }^{3}$ H. H. Wills Physics Laboratory, University of Bristol, Bristol BS8 1TL, United Kingdom \\ ${ }^{4}$ Institute of Theoretical Physics, University Regensburg, 93040 Regensburg, Germany
}

(Received 2 October 2012; published 12 April 2013)

\begin{abstract}
The effect of electron-impurity scattering on momentum and spin relaxation times in graphene is studied by means of relativistic ab initio calculations. Assuming carbon and silicon adatoms as natural impurities in graphene, we are able to simulate fast spin relaxation observed experimentally. We investigate the dependence of the relaxation times on the impurity position and demonstrate that $\mathrm{C}$ or $\mathrm{Si}$ adatoms act as real-space spin hot spots inducing spin-flip rates about 5 orders of magnitude larger than those of in-plane impurities. This fact confirms the hypothesis that the adatom-induced spin-orbit coupling leads to fast spin relaxation in graphene.
\end{abstract}

DOI: 10.1103/PhysRevLett.110.156602

PACS numbers: 72.80.Vp, 71.70.Ej, 72.25.Rb, 73.22.Pr

One of the promising features of graphene [1] for spintronics applications [2] is the long spin lifetime expected from the weak spin-orbit coupling (SOC) [3]. In combination with robust electrical spin injection efficiency [4-12], slow spin relaxation would offer a way to graphene-based spintronics. However, nonlocal spin injection experiments [4,7-12] provide consistently short spin relaxation times of several hundred picoseconds. By contrast, ideal graphene is expected to have spin relaxation times of at least several microseconds [3]. The clarification of this discrepancy is very important for future applications of graphene.

The spin relaxation of conduction electrons in graphene can be of various origin. They include intrinsic spin-orbit effects and extrinsic spin-orbit fields mainly due to ripples [13-19] or the substrate [20,21]. The most reasonable results were obtained with models assuming that adatoms enhance the spin-orbit coupling locally [16,20-23]. For instance, it has been proposed that chemisorbed adatoms, such as hydrogen, can locally increase the spin-orbit fields due to $s p^{3}$ bonding [23,24]. However, there have been no first-principles calculations of the spin relaxation time yet to confirm these predictions.

In this Letter we report $a b$ initio studies of the impurityinduced scattering of the Dirac electrons in graphene. The calculations of both spin-conserving and spin-flip scattering rates are based on our approach recently developed and successfully used for bulk systems [25]. With this approach, adapted here for the slab geometry of graphene, we describe the spin relaxation time within the standard Elliott-Yafet spin relaxation mechanism [26]. This mechanism means that conventional scattering processes at impurities, boundaries, interfaces, and by phonons can change the spin state of an electron in the presence of SOC [2]. We deliberately focus on rather light impurities, namely, carbon and silicon being naturally present in graphene, and show that despite their weak atomic SOC they can give fast spin-flip rates at reasonable impurity densities. Our main finding is that these rates depend enormously on the impurity position. Particularly strong spin-flip scatterers are located on top of the bond between the host carbon atoms. We argue that these act as spatial spin hot spots, which are introduced in analogy to the known momentum-space spin hot spots [27]. As an extrinsic mechanism breaking space inversion symmetry and inducing strong spin-orbit fields locally, they can be responsible for the experimentally observed short spin relaxation times.

Our host system is a freestanding flat graphene sheet. To determine energetically favorable positions of the $\mathrm{C}$ and $\mathrm{Si}$ adatoms on graphene, we used the VASP code [28] within the density functional theory. Three different positions of an isolated adatom were simulated within a 24-atom graphene supercell: (i) on top of the graphene hollow site $(\mathrm{OH})$, (ii) on top of the bridge between two host carbon atoms (OB), and (iii) on top of the graphene site (OS). The determined out-of-plane distances of each adatom configuration as well as their energetics are shown in Fig. 1. For both carbon and silicon adatoms, the position OB is energetically preferable compared to the two others. This is in agreement with previous ab initio calculations [29].

Figure 1 also shows the charge density contour plots on a vertical plane intersecting the graphene bridge and the $\mathrm{C}$ adatom. A similar picture for the $\mathrm{Si}$ adatom in the $\mathrm{OB}$ position was presented in Ref. [29]. Although the C-C bond between the graphene atoms and the adatom is weaker than the in-plane graphene bond, the adatom induces a strong change of the charge density gradient. This is related to the gradient of the potential perturbation which defines the strength of the effective SOC, 


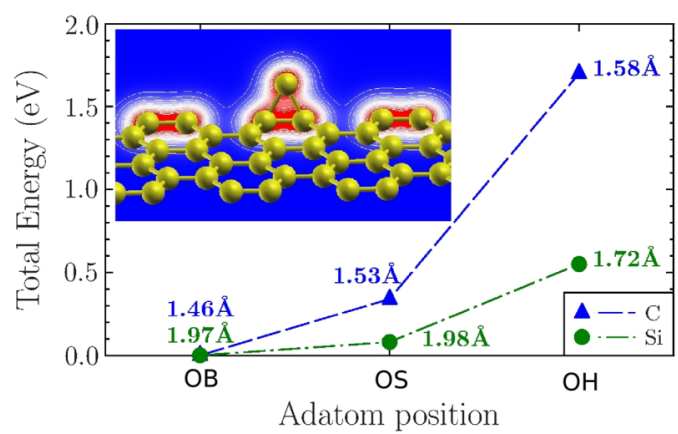

FIG. 1 (color online). Determined from fist-principles out-ofplane distances for $\mathrm{C}$ and $\mathrm{Si}$ adatoms with corresponding energetics. Inset: Charge distribution for $\mathrm{C}$ adatom on top of the bridge position in graphene.

$$
\hat{H}_{\mathrm{SO}}^{\mathrm{eff}} \sim \hat{\mathbf{s}} \cdot\left[\hat{\mathbf{p}} \times \nabla_{\mathbf{r}}(\Delta V)\right]=\hat{\mathbf{p}} \cdot\left[\nabla_{\mathbf{r}}(\Delta V) \times \hat{\mathbf{s}}\right],
$$

where $\hat{\mathbf{s}}$ and $\hat{\mathbf{p}}$ are the operators of the spin and the momentum of the electron, respectively. Here, $\Delta V(\mathbf{r})$ represents the change of the potential with respect to the ideal host system. As was discussed in Ref. [25], an enhancement of $\hat{H}_{\mathrm{SO}}^{\text {eff }}$, the change in the SOC induced by the impurity in the host system, is crucial for the considered spin relaxation mechanism.

The impurity at the energetically favorable OB position is our realization of a space inversion symmetry breaking adatom inducing, via $s p^{3}$ bonding [23], strong spin-orbit fields. For reference, we also calculate the scattering rates for the in-plane hollow site (IH) impurity position, known to be quite feasible for point defects in graphene [30]. This position preserves space inversion symmetry and does not induce strong spin-orbit fields. We will show that the corresponding spin-flip rates are orders of magnitude below the OB case.

The electronic structure of graphene was calculated by means of a relativistic Korringa-Kohn-Rostoker method [31]. Because of the presence of both space and time inversion symmetry of the graphene host, each electronic band is twofold degenerate having two Dirac spinor states $\Psi_{\mathbf{k}}^{+}$and $\Psi_{\mathbf{k}}^{-}$with opposite spin polarizations [31]. The momentum and spin relaxation times are obtained from the calculated microscopic transition probability $P_{\mathbf{k} \mathbf{k}^{\prime}}^{s s^{\prime}}$ expressed, similar to the case of bulk systems [25], via Fermi's golden rule

$$
P_{\mathbf{k} \mathbf{k}^{\prime}}^{s s^{\prime}}=\frac{2 \pi}{\hbar} n_{i} S\left|T_{\mathbf{k k}^{\prime}}^{s \mathbf{k}^{\prime}}\right|^{2} \delta\left(E_{k}-E_{k^{\prime}}\right)
$$

as discussed in the Supplemental Material [32]. Here $n_{i}$ denotes the impurity density, providing together with the sheet area $S$ the total number of impurities $n_{i} S$ in the system. The transition matrix [25]

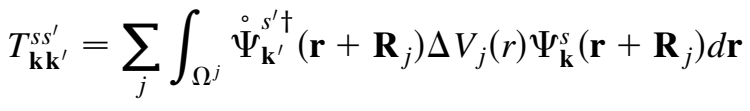

describes the transition amplitude for the scattering from the initial momentum state $\mathbf{k}$ and spin state $s$ to the corresponding final states $\mathbf{k}^{\prime}$ and $s^{\prime}$. To obtain this quantity, we use the approach of Refs. $[25,33]$ with technical details described in the Supplemental Material [32]. Taking the Fermi surface averages

$$
1 / \tau^{s s^{\prime}}=\left\langle 1 / \tau_{\mathbf{k}}^{s s^{\prime}}\right\rangle_{\mathbf{k}}, \quad 1 / \tau_{\mathbf{k}}^{s s^{\prime}}=\sum_{\mathbf{k}^{\prime}} P_{\mathbf{k} \mathbf{k}^{\prime}}^{s \prime^{\prime}}
$$

we obtain the momentum relaxation time $\tau$ and the spin relaxation time $T_{1}$ as

$\tau=\tau^{++}=\tau^{--}, \quad 1 / T_{1}=1 / \tau^{+-}+1 / \tau^{-+}=2 / \tau^{+-}$,

where we have used the symmetry relation $\tau^{-+}=\tau^{+-}$.

To calculate these quantities with Eqs. (2)-(5), we assume $n_{i}=2 \times 10^{12} \mathrm{~cm}^{-2}$, which corresponds to one impurity atom per 1000 unit cells, similar to estimations based on experimental data for charged impurities [34]. Such an impurity concentration provides the dilute limit for which Eq. (2) is valid. As mentioned above, our aim is to investigate the spin relaxation in graphene caused by the SOC enhanced by impurities. To follow this idea, we consider only nonmagnetic solutions of the impurity problem neglecting a small magnetization induced for some impurity positions, which would open an additional scattering path.

The main result of our work is shown in Table I, where the momentum and spin relaxation time provided by $\mathrm{C}$ and $\mathrm{Si}$ adatoms is presented in comparison to the related in-plane impurities. The calculations are performed at the energy of $0.12 \mathrm{eV}$ above the Dirac point. This relates to a carrier density of $\sim 2 \times 10^{12} \mathrm{~cm}^{-2}$ set by a gate voltage in experiments [4]. The corresponding experimental data are shown in the Table I as well. One can see that the in-plane impurity position (IH) yields reasonable values for $\tau$, with respect to the experimental data, for both impurity atoms. However, the corresponding spin relaxation time is on the microsecond scale, which is a common theoretical expectation for the spin relaxation caused by the intrinsic SOC in graphene [3]. Thus, such light impurities as $\mathrm{C}$ and $\mathrm{Si}$ atoms in the graphene sheet cannot modify the SOC significantly.

TABLE I. The momentum relaxation time $\tau$ and the spin relaxation time $T_{1}$ for $\mathrm{C}$ and $\mathrm{Si}$ impurities on top of the bridge (OB) and on the in-plane hollow site $(\mathrm{IH})$ positions. The results are shown for the spin polarization out of plane $\left(T_{1}^{\perp}\right)$ and in plane $\left(T_{1}^{\|}\right)$, which does not affect $\tau$. For comparison, the typical orders of magnitude for the experimentally observed momentum and spin relaxation times [4] are given.

\begin{tabular}{lccc}
\hline \hline Our theory & $\tau$ & $T_{1}^{\perp}$ & $T_{1}^{\|}$ \\
\hline OB $(\mathrm{C})$ & $300 \mathrm{fs}$ & $27 \mathrm{~ns}$ & $13 \mathrm{~ns}$ \\
IH (C) & $65 \mathrm{fs}$ & $130 \mu \mathrm{s}$ & $1.9 \mu \mathrm{s}$ \\
OB (Si) & $73 \mathrm{fs}$ & $210 \mathrm{ps}$ & $67 \mathrm{ps}$ \\
IH (Si) & $19 \mathrm{fs}$ & $39 \mu \mathrm{s}$ & $1.2 \mu \mathrm{s}$ \\
Experiment & $\sim 10 \mathrm{fs}$ & \multicolumn{2}{c}{$\sim 100 \mathrm{ps}$} \\
\hline \hline
\end{tabular}


On the other hand, the spin relaxation time becomes orders of magnitude shorter in the case of both $\mathrm{C}$ and $\mathrm{Si}$ impurities in the OB position. In particular, $\mathrm{Si}$ adatoms yield values of $T_{1}$ comparable to the experimental data. Consequently, for a fast spin relaxation a strong change of the potential in the out-of-plane direction caused by adatoms is important.

Remarkably, Si adatoms, which can be provided by the $\mathrm{SiO}_{2}$ substrate used in the experiment [4], also yield the momentum relaxation time close to the experimental data. However, the obtained $\tau$ is about 4 times larger for adatoms in comparison to impurities in plane. Thus, the momentum relaxation in graphene is more affected by in-plane impurities than by adatoms. By contrast, the contribution of the adatoms to the spin relaxation is incredibly enhanced with respect to other impurities. For instance, the relevant ratio $\tau / T_{1}$ for the spins out of plane is about $10^{-9}$ for $\mathrm{Si}$ impurities in the IH position while it is increased up to $\sim 10^{-3}$ for $\mathrm{Si}$ adatoms. This shows that adatoms play the role of spatial spin hot spots in analogy to the momentumspace spin hot spots [27].

The momentum scattering is practically not influenced by the SOC, which is confirmed by the fact that the calculated $\tau$ is unchanged by a rotation of the quantization axis. By contrast, the spin relaxation time has a strong anisotropy with respect to the spin direction, as shown in Table I. This can be understood applying Eq. (1) and taking into account that for the considered spin scattering the gradient of the potential in the $z$ direction (out of plane) is crucial, which follows simply from the comparison of adatoms and impurities in plane. According to Eq. (1), the effective SOC provided by $\Delta_{z}(\Delta V)$ should be larger for the spins pointing in the plane in comparison to the ones pointing out. This leads to a stronger spin-flip scattering for electrons with the spin in plane, as reflected in our results. However, it is opposite to an early experimental observation, where a $20 \%$ decrease in the spin relaxation time was found for electrons with spins perpendicular to the graphene plane [4]. This subtle point requires further systematic theoretical and experimental investigations. Nevertheless, it is clear that the presence of a substrate in experiments causes Rashba-type spin-orbit fields pointing in the graphene plane. These fields provide stronger relaxation of spins in the out-of-plane direction, since all of them are transverse to the spin-orbit fields. By contrast, for electrons injected in graphene with spins in a certain in-plane direction, only half of the Rashba-type spin-orbit fields have effectively transverse orientation. Thus, a simple estimation for such a mechanism gives $T_{1}^{\perp} / T_{1}^{\|} \approx \frac{1}{2}$ [2]. Taking into account this point, the $20 \%$ anisotropy observed in the experiment can be interpreted as a result of interference between the mechanism caused by adatoms and the one induced by the substrate.

The Elliott-Yafet spin relaxation mechanism means a linear relation between $T_{1}$ and $\tau$ [26]. Such a correlation is nicely demonstrated in the upper panel of Fig. 2. Here, the

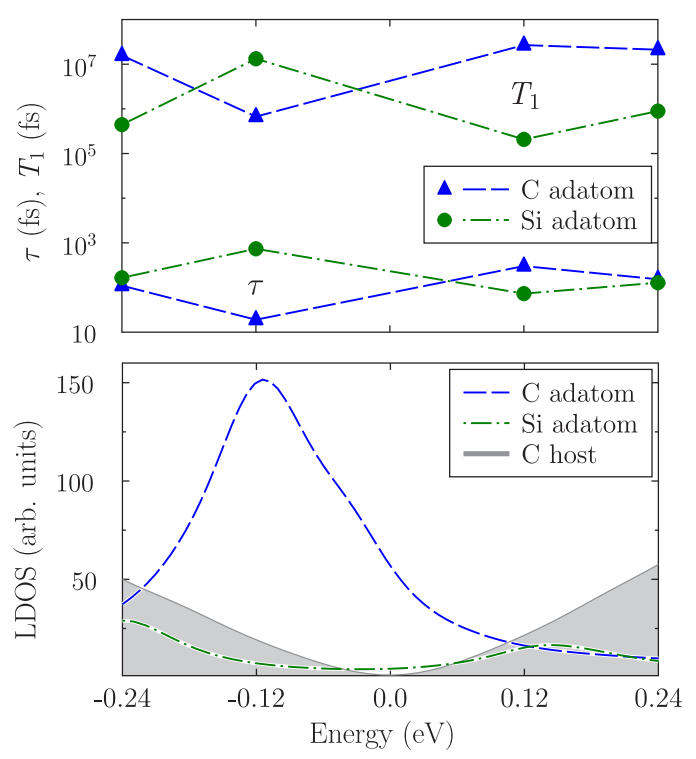

FIG. 2 (color online). Upper panel: Spin and momentum relaxation time, $T_{1}$ and $\tau$, caused by $\mathrm{C}$ and $\mathrm{Si}$ adatoms (for spins in the out-of-plane direction). The lines are to guide the eyes. Lower panel: Local density of states of the adatoms and C host atom (the latter one is multiplied by the factor of 200). The energy is counted with respect to the Dirac point.

energy dependence of the scattering properties is caused by the impurity states. This is shown in the lower panel of Fig. 2, where the local density of states (LDOS) of the C and $\mathrm{Si}$ adatoms is presented in comparison to the LDOS of a $\mathrm{C}$ host atom. In addition, a silicon atom, being isovalent to a carbon one, has a larger atomic number. This causes an increased SOC strongly affecting the spin relaxation time. The energy dependence of our results shown in the upper panel of Fig. 2 does not follow the experimental findings, where $\tau$ and $T_{1}$ as functions of the energy reflect the linear dispersion relation well known for graphene $[6,8-10,12]$. To clarify this point, further investigations are required. In particular, consideration of other reasonable adatoms, such as hydrogen, is very desirable.

It is important to mention that our method allows one to investigate possible scenarios with more than one type of impurities in experimental samples. In the dilute limit of noninteracting scattering centers, the relaxation times can be calculated in analogy to Matthiessen's rule as $1 / \tau=$ $1 / \tau^{\alpha}+1 / \tau^{\beta}$ and $1 / T_{1}=1 / T_{1}^{\alpha}+1 / T_{1}^{\beta}$, where the symbols $\alpha$ and $\beta$ label two different types of defects. For example, let us assume $\mathrm{Si}$ impurities to be present at the same time in the IH and OB positions. Then, the in-plane impurities can provide the momentum relaxation, while adatoms are responsible for the spin relaxation. Applying Matthiessen's rule and using the results of Table I, we obtain the momentum relaxation time as $\tau=15 \mathrm{fs}$ and the spin relaxation time as $T_{1}=210 \mathrm{ps}$ for spins in the out-of-plane direction. Both values are close to the experimental data, but they are provided from two different 
impurities. This example can simply explain the experiment of Ref. [7] with Au doping of graphene, where no linear relation between $\tau$ and $T_{1}$ was obtained. In fact, for each type of defect the relation, required by the ElliottYafet mechanism, can still be valid. However, owing to different dominating contributions for $\tau$ and $T_{1}$, this relation was not observed in the experiment. Such a situation is important to understand the variety of experiments showing a nonlinear relation between the two relaxation times, like it was observed in the bilayer graphene at low temperature [8-10,12]. Normally, one assumes that the Dyakonov-Perel mechanism is dominant in this case. However, our study shows that the absence of the proportionality between $\tau$ and $T_{1}$ can be explained within the Elliott-Yafet mechanism as well.

We should also mention that the adatom-induced spin relaxation in graphene cannot be properly described by the phase-shift model [25], which provides $T_{1}$ of 68 and $47 \mu \mathrm{s}$ for Si impurities in the OB and the IH position, respectively. The reason is that this model holds for atomic SOC but fails for SOC induced by strong covalent bonds. Thus, the complete calculations, based on Eqs. (2)-(5), are required for a proper theoretical study of the spin relaxation caused by adatoms on graphene.

In summary, we have demonstrated from first principles that space inversion symmetry breaking adatoms on graphene act as spatial spin hot spots, providing spin relaxation rates several orders of magnitude larger than symmetry conserving impurities. Thus, on the level of $a b$ initio calculations we have proven that the spin-orbit coupling induced by adatoms causes drastically reduced spin relaxation times comparable to experimental data.

This work was supported by the Deutsche Forschungsgemeinschaft (DFG) via SFB 762 and SFB 689 projects. In addition, M. G. acknowledges financial support from the DFG via a research fellowship (GR3838/1-1). We also acknowledge the technical support of Peter Zahn with respect to Fermi surface integration.

*dfedorov@mpi-halle.mpg.de

[1] A. K. Geim and K.S. Novoselov, Nat. Mater. 6, 183 (2007); A.H. Castro Neto, Science 332, 315 (2011); D. A. Abanin et al., Science 332, 328 (2011).

[2] I. Žutič, J. Fabian, and S. Das Sarma, Rev. Mod. Phys. 76, 323 (2004); J. Fabian, A. Matos-Abiague, C. Ertler, P. Stano, and I. Žutič, Acta Phys. Slovaca 57, 565 (2007).

[3] D. Pesin and A.H. MacDonald, Nat. Mater. 11, 409 (2012).

[4] N. Tombros, C. Józsa, M. Popinciuc, H. T. Jonkman, and B. J. van Wees, Nature (London) 448, 571 (2007); N. Tombros, S. Tanabe, A. Veligura, C. Józsa, M. Popinciuc, H. T. Jonkman, and B. J. van Wees, Phys. Rev. Lett. 101, 046601 (2008).

[5] M. Ohishi, M. Shiraishi, R. Nouchi, T. Nozaki, T. Shinjo, and Y. Suzuki, Jpn. J. Appl. Phys. 46, L605 (2007).
[6] C. Józsa, T. Maassen, M. Popinciuc, P. J. Zomer, A. Veligura, H. T. Jonkman, and B. J. van Wees, Phys. Rev. B 80, 241403(R) (2009).

[7] K. Pi, W. Han, K. M. McCreary, A. G. Swartz, Y. Li, and R. K. Kawakami, Phys. Rev. Lett. 104, 187201 (2010).

[8] T.-Y. Yang et al., Phys. Rev. Lett. 107, 047206 (2011).

[9] W. Han and R. K. Kawakami, Phys. Rev. Lett. 107, 047207 (2011).

[10] A. Avsar et al., Nano Lett. 11, 2363 (2011).

[11] S. Jo, D.-K. Ki, D. Jeong, H.-J. Lee, and S. Kettemann, Phys. Rev. B 84, 075453 (2011).

[12] W. Han, K. M. McCreary, K. Pi, W.H. Wang, Y. Li, H. Wen, J.R. Chen, and R. K. Kawakami, J. Magn. Magn. Mater. 324, 369 (2012).

[13] D. Huertas-Hernando, F. Guinea, and A. Brataas, Phys. Rev. B 74, 155426 (2006).

[14] B. Dora, F. Muranyi, and F. Simon, Europhys. Lett. 92, 17002 (2010).

[15] F. Simon, F. Muranyi, and B. Dora, Phys. Status Solidi B 248, 2631 (2011)

[16] V. K. Dugaev, E. Ya. Sherman, and J. Barnas, Phys. Rev. B 83, 085306 (2011).

[17] S. Fratini, D. Gosalbez-Martinez, P. Merodio Camara, and J. Fernandez-Rossier, arXiv:1202.6216.

[18] P. Zhang, Y. Zhou, and M. W. Wu, J. Appl. Phys. 112, 073709 (2012).

[19] J.-S. Jeong, J. Shin, and H.-W. Lee, Phys. Rev. B 84, 195457 (2011).

[20] C. Ertler, S. Konschuh, M. Gmitra, and J. Fabian, Phys. Rev. B 80, 041405(R) (2009).

[21] P. Zhang and M. W. Wu, Phys. Rev. B 84, 045304 (2011); New J. Phys. 14, 033015 (2012).

[22] H. Ochoa, A. H. Castro Neto, and F. Guinea, Phys. Rev. Lett. 108, 206808 (2012).

[23] A. H. Castro Neto and F. Guinea, Phys. Rev. Lett. 103, 026804 (2009).

[24] D. Huertas-Hernando, F. Guinea, and A. Brataas, Phys. Rev. Lett. 103, 146801 (2009).

[25] M. Gradhand, D. V. Fedorov, P. Zahn, and I. Mertig, Phys. Rev. B 81, 020403(R) (2010).

[26] R. J. Elliott, Phys. Rev. 96, 266 (1954); Y. Yafet, in Solid State Physics, edited by F. Seitz and D. Turnbull (Academic, New York, 1963), Vol. 14.

[27] J. Fabian and S. Das Sarma, Phys. Rev. Lett. 81, 5624 (1998); 83, 1211 (1999).

[28] G. Kresse and J. Hafner, Phys. Rev. B 49, 14251 (1994).

[29] E. Aktürk, C. Ataca, and S. Ciraci, Appl. Phys. Lett. 96, 123112 (2010).

[30] P. T. Araujo, M. Terrones, and M.S. Dresselhaus, Mater. Today 15, 98 (2012).

[31] M. Gradhand, M. Czerner, D. V. Fedorov, P. Zahn, B. Yu. Yavorsky, L. Szunyogh, and I. Mertig, Phys. Rev. B 80, 224413 (2009).

[32] See Supplemental Material at http://link.aps.org/ supplemental/10.1103/PhysRevLett.110.156602 for a more detailed description of the computational scheme.

[33] I. Mertig, Rep. Prog. Phys. 62, 237 (1999).

[34] Y.-W. Tan, Y. Zhang, K. Bolotin, Y. Zhao, S. Adam, E. H. Hwang, S. Das Sarma, H. L. Stormer, and P. Kim, Phys. Rev. Lett. 99, 246803 (2007). 